the SMR for patients with definite epilepsy was 3.0. It was highest during the first year after diagnosis (5.1), and declined to 2.5 at 3 years and 1.3 at 5 years. The SMR was highest in cases of remote symptomatic epilepsy (4.3), it was 2.9 with acute symptomatic epilepsy, 1.6 with idiopathic epilepsy, and 0 for febrile seizures. The commonest causes of death were pneumonia, cancer, and stroke. (Cockerell OC et al. Mortality from epilepsy: results from a prospective population-based study. Lancet Oct 1, 1994;344:918-921). (Respond: Dr OC Cockerell, Chalfont Centre for Epilepsy, Chalfont St Peter, Bucks SL9 ORJ, UK).

COMMENT. The high mortality in patients with epilepsy was due mainly to the underlying cause, although idiopathic epilepsy itself carries an increased risk. The death rate was highest in the first year after diagnosis, the result of associated diseases such as stroke and tumor, and then decreased progressively. Heart disease was not a factor. An increased incidence of suicide previously reported in patients with epilepsy was not confirmed in this study.

Heautoscopy, epilepsy, and suicide. A 21 year-old man with complex partial seizures who tried to commit suicide during the experience of heautoscopy is reported from the University Hospital, Zurich, Switzerland. As the classic doppelganger experience, heautoscopy, the reduplicative hallucination of one's own person, combines features of autoscopy (a mirror reflection of one's body) and an out of body experience (illusion of physical separation from one's own body). Seizures associated with heautoscopy usually originate in parietal or deep temporal foci. (Brugger P et al. I Neurol Neurosurg Psychiatry July 1994;57:838).

\title{
VISUALLY INDUCED SEIZURES
}

Photosensitivity and pattern sensitivity were evaluated in 67 reactive epileptic children, aged 4 - 19 years, at Universita "La Sapienza," Rome, Italy. Fifty-one percent showed sensitivity to both light and pattern, 33\% showed photosensitivity, and $16 \%$ were pattern sensitive. Pattern sensitive patients without photosensitivity had a higher incidence of focal symptomatic epilepsies, neurologic abnormalities, and focal EEGs. The EEG in pattern sensitive children showed focal polyspikes, spikes, slow or sharp waves in occipital regions, whereas photosensitive patients had more frequent generalized polyspike-wave and spike-wave complexes in the EEG. (Brinciotti $M$ et al. Pattern sensitivity and photosensitivity in epileptic children with visually induced seizures. Epilepsia July/August 1994;35:842-849). (Reprints: Dr M Brinciotti, Instituto di Neuropsichiatria Infantile, Universita "La Sapienza," Via dei Sabelli 108, 00185 Rome, Italy).

COMMENT. Patients with pattern sensitivity are at risk of focal, symptomatic seizures and neurologic abnormalities, whereas those with photosensitivity have generalized seizure patterns.

Video-game epilepsy. Kasteleijn-Nolst Trenite DGA, of the Instituut voor Epilepsiebestrijding, Heemstede, the Netherlands, comments on video-game epilepsy (Lancet Oct 22, 1994;344:1102-3). 50 cases have been published world wide. Mean age is 13 years, and $75 \%$ are male. One-third had a prior spontaneous or visually induced non-video-game seizure, and $50 \%$ showed epileptiform EEG discharges with photic stimulation. 
Only $5 \%$ of epileptic patients in general are sensitive to photic stimulation. The video game is a specific provocative factor in predisposed "photosensitive" epileptic patients. These patients are also sensitive to television (40\%), flickering sunlight (35\%), disco lights (25\%), and striped patterns such as venetian blinds and escalators (10\%). In video game epilepsy, precipitating factors might include color, flashing lights or patterns, the cognitive content, and lack of sleep. Studies in the Netherlands show that viewing a "flashing" program on a $50 \mathrm{~Hz}$ television in close proximity to the set is the most provocative stimulus. A video game is more provocative than a picture with less color and movement. Children should be kept at a distance of at least 2 m from a television screen. (see Ped Neur Briefs Sept 1994).

\section{EPILEPSY AND HYPERKINETIC BEHAVIOR}

A 4-year-old boy with benign partial epilepsy and hyperkinetic behavior between seizures is reported from Sapporo Medical University, Japan. Hyperactivity was noted at age 3, and seizures began at 4 years 6 months. Attacks consisted of a terrified expression, crouching, and rubbing his forehead on the floor. They occurred in sleep and awake. An ictal EEG in sleep showed theta rhythm, predominant over the left hemisphere, followed by voltage depression, but no spike and wave complexes. Both seizures and hyperkinetic behavior responded to carbamazepine. The epilepsy was characterized as benign partial epilepsy with affective symptoms. (Wakai $S$ et al. Benign partial epilepsy with affective symptoms: Hyperkinetic behavior during interictal periods. Epilepsia July/Aug 1994;35:810-812). (Reprints: Dr S Wakai, Dept Pediatrics, Sapporo Medical University, School of Medicine, South 1 West 16, Chuo-ku, Sapporo, 060, Japan).

COMMENT. Behavioral and emotional disorder as a form of epilepsy is a controversial topic, and the response to antiepileptic drugs in treatment is not proof of epilepsy. This patient tends to support the concept of a specific epileptic syndrome, BPEAS, but the EEG evidence could be more convincing.

The following study provides evidence against the concept of a so-called "masked epilepsy" in some hyperkinetic children. A comparison of the emotional and behavioral problems of 53 children, aged 6-12 years, with epileptiform EEG discharges and those of children without this EEG abnormality showed no significant differences, in a study at the Tokyo Medical and Dental University; the Nihon University; Asai Hospital, Chiba; and National Center for Neurology and Psychiatry, Kodaira, Japan. The authors conclude that the emotional and behavioral problems are coincidental and not directly related to the epileptiform discharges. (Okubo Y et al. Epileptiform EEG discharges in healthy children: Prevalence, emotional and behavioral correlates, and genetic influences. Epilepsia July/Aug 1994;35:832-841). 